\title{
Transition metal sulfur chemistry and its relevance to molybdenum and tungsten enzymes
}

\author{
Edward I. Stiefel \\ Exxon Research and Engineering Co., Clinton Township, Rt 22E, Annandale, NJ \\ 08801
}

\begin{abstract}
Molybdenum or tungsten is present at the active sites of over 30 distinct enzymes. The Mo enzyme nitrogenase, with its unique polynuclear metal sulfide clusters, falls in a class by itself. All other Mo and W enzymes are mononuclear, with pterin-ene-dithiolate coordination. Recent $x$-ray crystallographic results on several of these enzymes confirm the potential additional presence of oxo (one or two), sulfido, cysteine, selenocysteine, serine, aquo/hydroxo, and/or a second dithiolene in the various metal coordination spheres. The rich chemistry of multisulfur transition metal systems admits ligand redox, internal electron transfer, and 'intermediate' redox states. This redox flexibility may facilitate coupled proton/electron transfer and/or oxo transfer mechanisms, which are effectively exploited by $\mathrm{Mo}$ and $\mathrm{W}$ enzymes. In the case of nitrogenase, a hypothesis is put forward that the relatively isolated heavy atom grouping at the FeMoco active site allows that site to become locally 'hot' during turnover, thereby facilitating activation of the recalcitrant dinitrogen molecule.
\end{abstract}

\section{MOLYBDENUM AND TUNGSTEN ENZYMES}

Molybdenum has been recognized as a requirement for bacterial nitrogen fixation for over 60 years (ref. 1) and as an essential element for animals for over 40 years (ref. 2). In the last 20 years (ref. 3,4), tungsten has joined its congener molybdenum as a component of redox enzymes (ref. 5,6). The molybdenum and tungsten enzymes have been extensively reviewed in recent years (ref. 5-18).

Nitrogenase, the enzyme responsible for nitrogen fixation, falls in a class by itself. Its metalloclusters, the $\mathbf{P}$ cluster and iron molybdenum cofactor, FeMoco, are polynuclear metal sulfide groupings arrayed to catalyze the multielectron reduction of the dinitrogen molecule (ref. 7,8,12,16,19). The FeMoco active site is illustrated in Fig. 1.

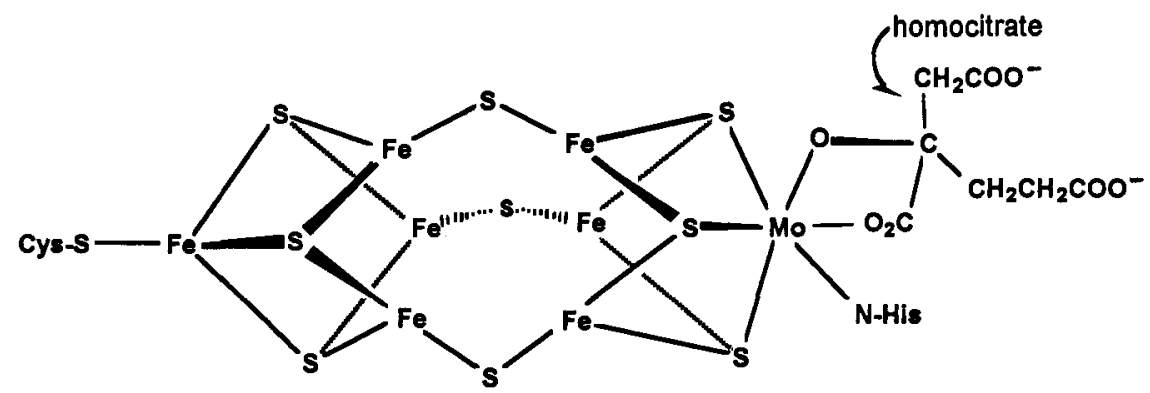

Fig. 1 The iron molybdenum cofactor of the iron molybdenum protein of nitrogenase. Note that the Fe7S9Mo cluster is only covalently linked to the protein at its terminal Mo and Fe atoms (ref. 12,19). 
The remaining molybdenum and tungsten enzymes, constituting over 30 different proteins, each have a mononuclear metal site with a specific pterin-ene-dithiolate coordination (ref. 5,6,10,11,13-18). This center, called Moco in molybdenum enzymes, is shown with its known variations in Fig. 2.

In all types of molybdenum and tungsten enzymes, the coordination sphere of the group VI element contains at least three of the heavier chalcogenide donors, i.e., usually three or more sulfur atoms but sometimes a lone selenium donor along with the sulfur atoms. These donors are disposed in the metal coordination sphere in adjacent positions so as to admit the possibility of significant interaction and, possibly, redox reactions of the types discussed below.

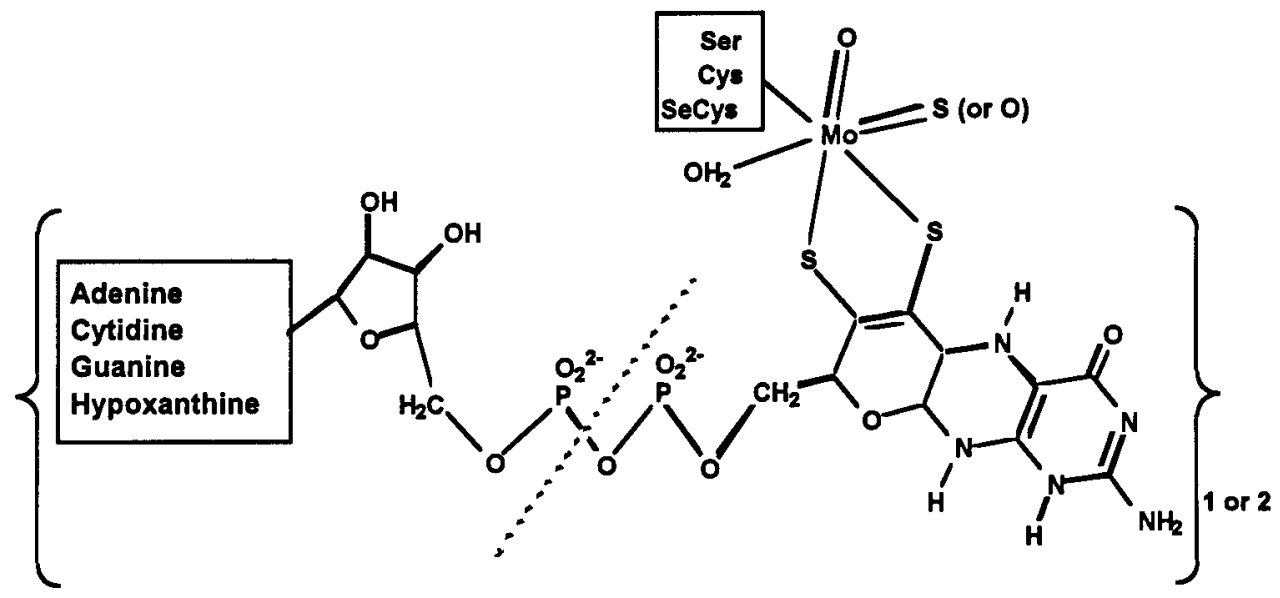

Fig. 2 The pterin-ene-dithiolate complex of molybdenum which constitutes the molybdenum cofactor (Moco), or when tungsten substitutes for molybdenum, the tungsten cofactor (Wco). The cofactor may have one or two dithiolene ligands, zero, one, or two oxo ligands and, in certain cases, a sulfido, serine, cysteine, selenocysteine, or aquo/hydroxo ligand. In some cases, a dinucleotide form of the ligand is present as shown in the material to the left of the dotted line.

\section{$\underline{\text { X-ray structures of Mo enzymes }}$}

The crystal structure of the nitrogenase proteins (ref. 12,19) has revealed the unique $P$ and FeMoco metalloclusters and their arrangement in the protein. Recently, the complex of the iron protein with the iron molybdenum protein has also been crystallographically elucidated (ref. 20). It is beyond the scope of this brief survey to describe the nitrogenase centers in any detail. However, we point out that FeMoco, shown in Fig. 1, is highly unusual with six low-coordinate iron atoms at its center and covalent connection to the protein at only two atoms at the extremities of the protein. Notwithstanding the details revealed by the crystallography (ref. 12,19,20) and despite the large numbers of elegant mechanistic studies that have been reported (ref. 7), we still do not understand the nature of dinitrogen activation and reduction.

In contrast to the situation with nitrogenase, are the recent determinations of crystal structures of the tungsten enzyme aldehyde ferredoxin oxidoreductase from Pyrococcus furiosus (ref. 21) and the molybdenum enzymes: aldehyde oxidoreductase from Desulfovibrio gigas (ref. 22,23); DMSO reductase from Rhodobacter sphaeroides (ref. 24) and Rhodobacter capsulatus (ref. 25,26) (three separate reports); formate dehydrogenase from Escherichia coli (ref. 27); and sulfite oxidase from chicken liver (ref. 28). In these structures, the mononuclear Mo or $\mathrm{W}$ sites display structural features that were previously inferred from spectroscopic and mechanistic studies, and provide details and distinctions not previously realized. In contrast to nitrogenase, the findings were immediately, albeit not unequivocally, interpretable in terms of mechanistic possibilities.

In the tungsten enzyme ferredoxin aldehyde oxidoreductase, crystallography unexpectedly revealed (ref. 21) the presence of two dithiolene ligands, both of which contain a three-ring dihydropyranopterin ligand (see Fig. 1). In contrast, the molybdenum aldehyde oxidoreductase was confirmed (ref. 23) to possess a 
Mo(VI) oxo sulfido mono pterin-dithiolene site (that had been deduced from EXAFS studies), which converts to a mono oxo sulfhydryl site on reduction by substrate to the Mo(IV) state (ref. 26). The three DMSO reductase structures (ref. 24-26) agree that each $\mathrm{Mo}(\mathrm{VI})$ is coordinated by a serine but reveal differences in the number of oxo groups (one vs. two), in whether the two dithiolene ligands are both coordinated to the Mo center, and in the redox state of one of them. Possibly, the reported structural differences are indicative of a molybdenum site that is structurally and electronically fluid, which may contribute to its catalytic activity. In formate dehydrogenase (ref. 27), two dithiolenes and a single selenocysteine ligand are bound to the Mo. In sulfite oxidase, a cysteine, one dithiolene and one or two oxo ligands are bound to the Mo center (ref. 28,29).

The crystallography reveals that previous formulations of reactivity in Mo and W enzymes in terms of oxygen atom transfer or proton electron transfer reactions are quite reasonably accommodated by the active site structures.

\section{MOLYBDENUM AND TUNGSTEN CHALCOGENIDE CHEMISTRY}

The presence of two or more sulfur (or sulfur and selenium) atoms in adjacent positions suggests the possibility of ligand redox processes. Such processes are illustrated in molybdenum and tungsten chalcogenide chemistry. We portray some of the potential changes by briefly reviewing chemistry of tetrachalcogenidometallate ions. Ligand redox is illustrated by the reaction of $\mathrm{WS}_{4}{ }^{2-}$ with disulfides of 1,1-dithiolate ligands, while internal redox trends are illustrated by reactions of $\mathrm{MoS}_{4}{ }^{2-}, \mathrm{WSe}_{4}{ }^{2-}, \mathrm{VS}_{4}{ }^{3-}$, or $\mathrm{ReS}_{4}{ }^{-}$with the same disulfides.

\section{Ligand redox}

The reaction of tetrathiotungstate with thiuram disulfide effects reduction of the thiuram disulfide to the dithiocarbamate, and oxidation of bound sulfide ligands on tungsten to bound disulfide (ref. 30). This reaction is illustrated in equation (1) where $\mathbf{R}=$ small alkyl group, e.g., methyl or ethyl. Here the tungsten is in the VI state of oxidation in both the starting material and in the product.

$$
\mathrm{WVIS}_{4}{ }^{2-}+\mathrm{R}_{2} \mathrm{NC}(\mathrm{S}) \mathrm{S}-\mathrm{SC}(\mathrm{S}) \mathrm{NR}_{2} \rightarrow \mathrm{WVI}\left(\eta_{2}-\mathrm{S}_{2}\right)\left(\mathrm{S}_{2} \mathrm{CNR}_{2}\right)_{2}
$$

In this redox reaction, the organic disulfide is the oxidant, which is reduced to form the two dithiocarbamate ligands that are coordinated to $\mathrm{W}$ in the product. Two of the sulfido ligands on tungsten are oxidized to give a disulfido $\left(\mathrm{S}_{2}{ }^{2-}\right)$ group, which remains coordinated to the tungsten. This process involves solely ligand redox as the oxidation state of the tungsten does not change.

A significant number of ligand redox processes occur in multisulfur molybdenum sites. Included among these are redox processes that involve as many as six electrons per molybdenum (ref. 31,32), which, nevertheless, occur without changing the oxidation state of the metal.

\section{Internal electron transfer}

In contrast to the reactions of tetrathiotungstate, the reactions of tetrathiomolybdate with the same thiuram disulfides, equation (2), give exclusively a product in which Mo is in the oxidation state V (ref. 30).

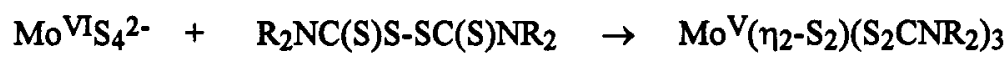

In this case, the molybdenum has been reduced by coordinated sulfide, which is oxidized to a disulfide. The remaining redox equivalents necessary to oxidize the sulfur come from the external oxidant, the thiuram disulfide. A key feature of this reaction is the internal electron transfer that occurs between the coordinated $\mathrm{S}^{2-}$ and the $\mathrm{Mo}(\mathrm{VI})$ ion. 
The comparison of reactions (1) and (2) raises the question: Why does the tungsten complex react by purely ligand redox while the molybdenum complex undergoes an internal electron transfer process? Clearly, molybdenum is generally more easily reduced than is tungsten. One manifestation of this trend is the position of the lowest energy ligand-to-metal charge-transfer (LMCT) transition in the electronic absorption spectrum. This transition occurs at lower energy in $\mathrm{MoS}_{4}{ }^{2-}$ (at $21,400 \mathrm{~cm}^{-1}$ ) than in $\mathrm{WS}_{4}{ }^{2-}$ (at $25,500 \mathrm{~cm}^{-1}$ ).

If the trend in LMCT correlates with the tendency toward internal redox reactions then there are other tetrathiometallates, such as $\mathrm{ReS}_{4}{ }^{-}$and $\mathrm{VS}_{4}{ }^{3-}$, that would be predicted to undergo internal electron transfer based on the low energy of their LMCT. Indeed, both rhenium and vanadium tetrathiometallates react dramatically in internal redox reactions (ref. 33-35).

An interesting test of the correlation between reactivity and LMCT involves the ion $\mathrm{WSe}_{4}{ }^{2-}$, whose LMCT comes at almost exactly the same energy as that of $\mathrm{MoS}_{4}{ }^{2-}$.

$$
\mathrm{WVISe}_{4}{ }^{2-}+\mathrm{R}_{2} \mathrm{NC}(\mathrm{S}) \mathrm{S}-\mathrm{SC}(\mathrm{S}) \mathrm{NR}_{2} \rightarrow \mathrm{WV}^{\mathrm{V}}\left(\eta_{2}-\mathrm{Se}_{2}\right)\left(\mathrm{S}_{2} \mathrm{CNR}_{2}\right)_{3}
$$

The reaction of the tetraselenotungstate shown in equation (3) exactly parallels that of the tetrathiomolybdate ion (ref. 36), again illustrating the utility of the correlation with the LMCT trends.

Interestingly, the first reported tungsten enzyme (ref. 3,4) also requires selenium for full activity. It is possible that nature chooses particular combinations of molybdenum or tungsten and sulfur or selenium to achieve needed redox or chemical reactivity. Other factors being equal, the tungsten/selenium pair may closely resemble the far more enzymatically common molybdenum/sulfur pair.

\section{The trithiolene-dithiolene conversion}

In studies of the molybdenum cofactor isolated from a variety of molybdenum enzymes, Rajagopalan and coworkers (ref. 15,37) identified a number of oxidized derivatives. Among these is a pterin with a fourcarbon side chain in which the first two carbon atoms are triply bound. Such pterin acetylenes were chemically synthesized by Taylor and coworkers (ref. 38) to confirm their structure. It seemed possible that reaction of the acetylene with a polysulfide molybdenum center could yield directly a pterin-enedithiolate structure, a potentially useful step in the total chemical synthesis of the molybdenum cofactor. Indeed, appropriate pterin- and related quinoxaline-acetylenes react (ref. 39,40) with mononuclear polysulfide centers according to the reaction shown in equation (4), $R=$ pterin or quinoxaline.

$$
\left(\eta{ }_{5}-\mathrm{C}_{5} \mathrm{H}_{5}\right)_{2} \mathrm{MoS}_{4}+\mathrm{RC} \equiv \mathrm{CC}(\mathrm{O}) \mathrm{CH}_{3} \rightarrow\left(\eta_{5}-\mathrm{C}_{5} \mathrm{H}_{5}\right)_{2} \mathrm{Mo}\left\{\mathrm{SC}(\mathrm{R})=\mathrm{C}\left[\mathrm{C}(\mathrm{O}) \mathrm{CH}_{3}\right] \mathrm{S}_{2}\right\}
$$

Remarkably, the reaction leads to the production of a trithiolene chelate rather than the expected dithiolene structure. The sulfur-rich trithiolene is, however, easily converted to the corresponding dithiolene by various sulfur abstracting agents such as triphenyl phosphine as shown in equation (5).

$$
\left(\eta_{5}-\mathrm{C}_{5} \mathrm{H}_{5}\right)_{2} \mathrm{Mo}\left\{\mathrm{S}_{2} \mathrm{C}(\mathrm{R})=\mathrm{C}\left[\mathrm{C}(\mathrm{O}) \mathrm{CH}_{3}\right] \mathrm{S}\right\}+\mathrm{Ph}_{3} \mathrm{P} \rightarrow\left(\eta_{5}-\mathrm{C}_{5} \mathrm{H}_{5}\right)_{2} \mathrm{Mo}\left\{\mathrm{SC}(\mathrm{R})=\mathrm{C}\left[\mathrm{C}(\mathrm{O}) \mathrm{CH}_{3}\right] \mathrm{S}\right\}+\mathrm{Ph}_{3} \mathrm{PS}(5)
$$

The existence of the trithiolene ligand in this molybdenum IV complex and its facile conversion to the dithiolene suggests interesting interconversions that might occur in multi-sulfur molybdenum or tungsten sites. These are illustrated in Fig. 3. 


\section{Internal Redox Isomers}

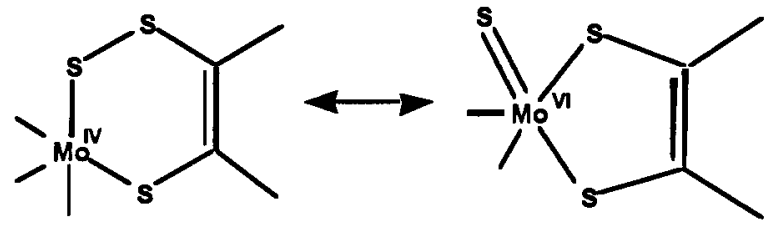

Mo(IV) Trithiolene

Mo(VI) Sulfido Dithiolene
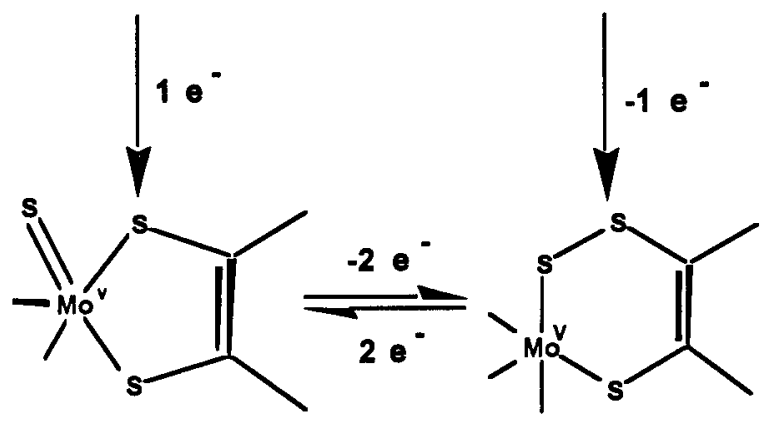

Mo(V) Sulfido Dithiolene

Mo(V) Trithlolene

Fig. 3 Hypothetical interconversion of sulfido dithiolene and trithiolene ligands that are suggested by studies of internal electron transfer and the chemical preparation of trithiolene and dithiolene complexes.

The figure shows the speculative scheme in which a sulfido dithiolene Mo(VI) is presented in the upper right. This unit is known to be present in the xanthine oxidase family of enzymes (ref. 10,11,22). Interestingly, the unit is a redox isomer of the trithiolene Mo(IV) site shown on the top left of the figure. The potential existence of both redox isomers allows seemingly paradoxical redox reactions to occur. For example, the right side (vertical) reaction shows that oxidation of the Mo(VI) site is possible yielding a trithiolene $\mathrm{Mo}(\mathrm{V})$ state in a reaction that involves induced internal electron transfer of the type described above. On the left side of the figure, the addition of an electron to the Mo(IV) complex can lead to the oxidation of the metal to $\mathrm{Mo}(\mathrm{V})$ provided that reduction of the disulfide linkage occurs concomitantly, i.e., an internal electron transfer reaction in the opposite sense. The bottom two species are both Mo(V) but differ by two electrons, which define the state of oxidation of the ligand(s) as either a sulfido dithiolene or a trithiolene. While there is no current evidence for trithiolene linkages in either Mo of W enzymes, the existence of these entities must be considered as possible during turnover.

\section{Intermediate redox states}

The previous subsections show that internal redox is possible between the metal and sulfur ligands while the ligands remain in the molybdenum or tungsten coordination sphere. The conversions are cleanly described in terms of electron or atom transfer processes and the oxidation states are unambiguously specified. A related situation occurs in certain transition metal sulfur complexes where contacts between nominally nonbonding sulfur atoms are sometimes far shorter that the S-S distance expected for sulfur atoms occupying adjacent coordination sites in the metal sphere.

One example is the complex $\mathrm{MoO}_{2}\left[\left(\mathrm{CH}_{3}\right)_{2} \mathrm{NCH}_{2} \mathrm{C}\left(\mathrm{CH}_{3}\right)_{2} \mathrm{~S}\right]_{2}$, wherein an unusual skew trapezoidal bipyramidal geometry is adopted (ref. 41,42 ). Here, the S-S distance is $2.76 \AA$, a value intermediate between a nonbonding S-S contact (expected to be around 3.2-3.3 $\AA$ ) and a fully bonded S-S linkage at around $2.1 \AA$. Another example is found in the complex $\mathrm{Mo}$ [tris(pyrazolylborate)]OS( $\left.\mathrm{S}_{2} \mathrm{PR}_{2}\right)$ where the contact between the nominally terminal sulfido linkage and one sulfur of the dithiophosphinate ligand is, at $2.396 \AA$, again intermediate between bonded and nonbonded distances (ref. 43). The existence of 
structures such as these can be viewed as involving partial electron transfer, wherein the ligand is partially oxidized and the metal partially reduced. However, a fully defined electron transfer has clearly not occurred. In these cases, while the metal oxidation state may be viewed in terms of one of the limiting formulation [Mo(VI) or $\mathrm{Mo}(\mathrm{IV})$ in the examples offered], in fact, the oxidation state may be difficult to unequivocally define in such cases.

Implications for the enzyme structures can be suggested. Thus, the surroundings of the metallo active site, i.e., the protein milieu, may favor one or another of the redox isomers, including either the limiting or intermediate forms in the electron transfer process. In view of the redox flexibility seen in the structures of DMSO reductase it seems reasonable to suggest that the enzymes have evolved to exploit particular electron distributions that facilitate substrate activation and conversion reactions. This flexibility may include the choice of ligand, metal, and redox isomer.

\section{The three oxidizable chalcogenides}

The types of redox processes discussed above require that the sulfur (or sulfur and selenium) ligands occupy adjacent (non trans) positions in the molybdenum or tungsten coordination sphere. For the two sulfur donors of each dithiolene ligand, this proximity is guaranteed by the chelating nature of the ligand. When more than one dithiolene ligand is present, at least one of the dithiolene ligands appears to bind strongly to the metal. Its role may involve activation of other groups in the coordination sphere, e.g., oxo, sulfido, selenocysteine, etc. to serve, for example, as proton acceptors or, in the case of the oxo ligand, as groups to be transferred to or from substrate. The second dithiolene, when present, may be less tightly bound and may be more able to participate in either short- or long-range redox processes. The presence of three or more oxidizable chalcogenide donors (S or Se) in each Mo or W coordination sphere may facilitate internal or ligand redox, which could be exploited in the functioning of particular enzymes.

\section{IS NITROGENASE FeMoco A HOT SITE?}

The structures of the Moco and Wco enzymes suggest or reinforce particular suggestions for their mechanisms of action. In contrast, the elucidation of the structure of FeMoco in nitrogenase has not led to easily testable mechanistic proposals for the action of the enzyme. Although theoretical studies have been performed (e.g., ref. 44), the lack of any spectroscopic or structural information about bound or intermediate forms has hampered full analysis.

One of the problems that nitrogenase and its FeMoco site must solve involves overcoming thermodynamic limitations for the generation of high energy intermediates [e.g., diazene $\left(\mathrm{N}_{2} \mathrm{H}_{2}\right)$, hydrazine $\left(\mathrm{N}_{2} \mathrm{H}_{4}\right)$, or other reduced forms] that may be part of the reaction pathway. One way to overcome this barrier is to strongly bind the intermediates and this possibility has been extensively discussed (ref. 16). A second possibility involves mobilization of sufficient reducing power (through the hydrolysis of ATP) such that the net energetics to reach the intermediate states is favorable.

We suggest an additional feature that may augment these possibilities. The overall reaction for the reduction of dinitrogen to ammonia is highly exergonic and exothermic. Equation (6),

$$
\mathrm{N}_{2}+16 \mathrm{H}_{2} \mathrm{O}+8 \mathrm{e}^{-}+16 \mathrm{MgATP} \rightarrow 2 \mathrm{NH}_{3}+\mathrm{H}_{2}+16 \mathrm{MgADP}+16 \mathrm{Pi}
$$

the generally accepted minimal reaction for nitrogenase (ref. 7,8), has a negative free energy change of about $-150 \mathrm{kcal} / \mathrm{mole}$. Interestingly, the formation of ammonia occurs with the mandatory co-production of dihydrogen. Equation (7) shows the reaction for the production of dihydrogen by nitrogenase,

$$
4 \mathrm{H}_{2} \mathrm{O}+2 \mathrm{e}^{-}+4 \mathrm{MgATP} \rightarrow \mathrm{H}_{2}+4 \mathrm{MgADP}+4 \mathrm{P}_{\mathrm{i}}
$$

which proceeds with $\Delta \mathrm{G}^{\circ} \sim-33 \mathrm{kcal} / \mathrm{mole}$. 
We suggest, as a speculative possibility, that, in nitrogenase, the hydrolysis of ATP and the transduction of that energy to $\mathrm{FeMoco}$ and/or the mandatory $\mathrm{H}_{2}$ evolution reaction may act to locally excite or heat the FeMoco site. This trapping of energy could allow formation of structural and/or electronic states other than those that have been identified spectroscopically or crystallographically (see Fig. 1). The high energy states could involve internal redox reactions that may be favored by the high-sulfur nature of the FeMoco site. Such reactions can generate additional reducing equivalents and/or open sites for substrate binding. For example, the formation of $\mathrm{S}_{2}{ }^{2-}$ from the oxidation of $2 \mathrm{~S}^{2-}$ can lead to two-electron reduction of the metal centers, which may facilitate the binding or reduction of dinitrogen.

The possibility that electronic or structural rearrangement may occur is augmented by the suggestion that the exothermic reactions of nitrogenase can locally heat the FeMoco site, raising its effective temperature such that it can bind and activate dinitrogen. It has been suggested, on theoretical grounds (ref. 45), that active sites containing heavy metals (and ligands) can be thermally isolated (decoupled) from the surrounding protein milieu. Such decoupling would be favored by the heaviness of the component atoms of the FeMoco cluster and the consequent low frequency of its vibrational and rotational motions. These low frequencies may effectively isolate FeMoco from the protein, whose motions are associated with higher frequency modes. The general notion is that the nitrogenase site may not be in thermal equilibrium with its protein surroundings during key phases of the nitrogen fixation process.

A curious feature of FeMoco is that it is only covalently linked to the protein at two points (at the extrema of the cluster). This unusual and minimal contact with the protein may further insulate the FeMoco site. Then, when $\mathrm{H}_{2}$ evolution occurs, driven by the hydrolysis of ATP, the energy released may be trapped in the cofactor. This excess energy could lead to different redox isomers, fluxionality of the cluster, and/or local heating. The isolation of the cluster may insure that the local excitation is trapped in FeMoco for a period of time sufficient to allow nitrogen fixation to occur. The indiscriminate reactivity of the activated nitrogenase system, which reduces a wide range of substrates (ref. 7), may be an indicator of the 'hot' and basically unstable nature of the active form of FeMoco. Indeed, if $\mathrm{N}_{2}$ or other reducible substrates are absent, the site does not remain in its activated form. Rather, it simply couples the electron and proton flows that it receives to produce additional dihydrogen.

If the FeMoco site is indeed 'hot', then the six-iron center of the cluster is vaguely reminiscent of the active $\mathrm{Fe}$ site in Haber process catalysts (ref. 46). In this industrially important process, high temperature (300$500^{\circ} \mathrm{C}$ ) and multi-iron coordination are key features of $\mathrm{N}_{2}$ activation. It would be ironic if the enzymic process resembles the Haber process in these respects.

\section{CONCLUSION}

The multi-sulfur sites present in all Mo and $\mathrm{W}$ enzymes may have evolved to exploit the redox capabilities inherent in such systems. The possibilities of ligand redox and internal electron transfer are supported by work on inorganic systems. These sulfur-based redox modes may supplement the metal-based electron transfer, coupled proton-electron transfer, and oxygen atom transfer processes that form the basis for substrate conversion. This great redox flexibility of the enzymic sites must be taken into account as probes of the structural, electronic structural, and mechanistic features of the Mo and W enzymes proceed. In the case of nitrogenase, the isolation of its FeMoco from the protein may allow the localized trapping of excess reaction energy to facilitate the dinitrogen reduction process.

\section{REFERENCES}

1. H. Bortels. Arch. Microbiol., 1, 333 (1930).

2. E. C. De Renzo, E. Kaleita, P. G. Heytler, J. J. Oleson, B. L. Hutchings and J. H. Williams. Arch. Biochem. Biophys., 45, 247-253 (1953).

3. L. G. Ljungdahl and J. R. Andreesen. Methods Enzymol., 53, 360-72 (1978).

4. L. G. Ljungdahl. Trends Biochem. Sci., 1, 63-5 (1976).

5. M. K. Johnson, D. C. Rees and M. W. W. Adams. Chem. Rev., 96, 2817-2839 (1996).

6. A. Kletzin and M. W. W. Adams. FEMS Microbiol. Rev., 18, 5-63 (1996).

7. B. K. Burgess and D. J. Lowe. Chem. Rev., 96, 2983-3011 (1996). 
8. R. R. Eady. Sci. Prog., 78, 1-17 (1995).

9. J. H. Enemark and C. G. Young, in Advances in Inorganic Chemistry, Vol. 40, Academic Press, New York, pp. 1-88 (1993).

10. R. Hille. J. Biol. Inorg. Chem., 1, $397-404$ (1996).

11. R. Hille. Chem. Rev., 96, 2757-2816 (1996).

12. J. B. Howard and D. C. Rees. Chem. Rev., 96, 2965-2982 (1996).

13. C. Kisker, H. Schindelin and D. C. Rees. Annu. Rev. Biochem., 66, 233-267 (1997).

14. R. S. Pilato and E. I. Stiefel, in Bioinorganic Catalysis (J. Reedijk, Ed.) Marcel Dekker, New York pp. 131-188 (1993).

15. K. V. Rajagopalan. ACS Symp. Ser., 535, 38-49 (1993).

16. E. I. Stiefel, D. Coucouvanis, and W. E. Newton, Editors, Molybdenum Enzymes, Cofactors, and Model Systems. ACS Symp. Ser., No. 535, ACS, Washington, D. C. (1993).

17. J. C. Wooton, R. E. Nicolson, J. M. Cock, D. E. Walters, J. F. Burke, W. A. Doyle and R. C. Bray. Biochim. Biophys. Acta., 1057, 157-185 (1991).

18. C. G. Young and A. G. Wedd, in Encyclopedia of Inorganic Chemistry (R. B. King, Ed.) John Wiley and Sons, New York, p. 2330 (1994).

19. J. Kim and D. C. Rees. Biochemistry, 33, 389-97 (1994).

20. H. Schindelin, C. Kisker, J. L. Schlessman, J. B. Howard and D. C. Rees. Nature, 387, 370-376 (1997).

21. M. K. Chan, S. Mukund, A. Kletzin, M. W. W. Adams and D. C. Rees. Science, 267, 1463-9 (1995).

22. R. Huber, P. Hof, R. O. Duarte, J. J. Moura, I. Moura, M.-Y. Liu, J. LeGall, R. Hille, M. Archer and R. M. J. Proc. Natl. Acad. Sci. USA, 93, 8846-8851 (1996).

23. M. J. Romao, M. Archer, I. Moura, J. J. G. Moura, J. LeGall, R. Engh, M. Schneider, P. Hof and R. Huber. Scence, 270, 1170-1176 (1995).

24. H. Schindelin, C. Kisker, J. Hilton, K. V. Rajagopalan and D. C. Rees. Science, 272, 1615-1621

25. A. S. McAlpine and S. Bailey. Internat. Union Crystallogr. Abstr., C, (1996).

26. F. Schneider, J. Loewe, R. Huber, H. Schindelin, C. Kisker and J. Knaeblein. J. Mol. Biol., 263, 53-69 (1996).

27. J. C. Boyington, V. N. Gladyshev, S. V. Khangulov, T. C. Stadtman and P. D. Sun. Science, 275, 1305-1308 (1997).

28. C. Kisker, H. Schindelin, D. C. Rees, A. Pacheco and J. N. Enemark. Submitted for publication, (1997).

29. G. N. George, R. M. Garrett, R. C. Prince and K. V. Rajagopalan. J. Am. Chem. Soc., 118, 8588-8592 (1996).

30. W.-H. Pan, T. R. Halbert, L. L. Hutchings and E. I. Stiefel. J. Chem. Soc., Chem. Commun., $927-9$ (1985).

31. K. F. Miller, A. E. Bruce, J. L. Corbin, S. Wherland and E. I. Stiefel. J. Am. Chem. Soc, 102, 5102-4 (1980).

32. T. R. Halbert, K. McGauley, W.-H. Pan, R. S. Czernuszewicz and E. I. Stiefel. J. Am. Chem. Soc., 106, 1849-51 (1984).

33. T. R. Halbert, L. L. Hutchings, R. Rhodes and E. I. Stiefel. J. Am. Chem. Soc., 108, 6437-8 (1986).

34. L. Wei, T. R. Halbert, H. H. Murray III and E. I. Stiefel. J. Am. Chem. Soc,, 112, 6431-3 (1990).

35. H. H. Murray, L. Wei, S. E. Sherman, M. A. Greaney, K. A. Eriksen, B. Carstensen, T. R. Halbert and E. I. Stiefel. Inorg. Chem., 34, 841-53 (1995).

36. Y. Gea, M. A. Greaney, C. L. Coyle and E. I. Stiefel. J. Chem. Soc., Chem. Commun., 160-1 (1992).

37. K. V. Rajagopalan and J. L. Johnson. J. Biol. Chem., 267, 10199 (1992).

38. E. C. Taylor, P. S. Ray and I. S. Darwish. J. Am. Chem. Soc., 111, 7664 (1989).

39. R. S. Pilato, K. A. Eriksen, M. A. Greaney, E. I. Stiefel, S. Goswami, L. Kilpatrick, T. G. Spiro, E. C. Taylor and A. L. Rheingold. J. Am. Chem. Soc., 113, 9372-4 (1991).

40. R. S. Pilato, K. Eriksen, M. A. Greaney, Y. Gea, E. C. Taylor, S. Goswami, L. Kilpatrick, T. G. Spiro, A. L. Rheingold and E. I. Stiefel. ACS Symp. Ser., 535, 83-97 (1993).

41. J. M. Berg, D. J. Spira, K. O. Hodgson, A. E. Bruce, K. F. Miller, J. L. Corbin and E. I. Stiefel. Inorg. Chem., 23, 341218 (1984).

42. E. I. Stiefel, K. F. Miller, A. E. Bruce, J. L. Corbin, J. M. Berg and K. O. Hodgson. J. Am. Chem. Soc., 102, 3624-6 (1980).

43. A. A. Eagle, L. J. Laughlin, C. G. Young and E. R. T. Tiekink. J. Am. Chem. Soc., 114, 9195-7 (1992).

44. I. Dance. J. Biol. Inorg. Chem., 1, 581-586 (1996).

45. M. Solc. J. Theor. Biol., 175, 57-61 (1995).

46. M. V. Twigg, Catalyst Handbook, Second Ed. Wolfe Publishing, Frome, England pp. 384-440 (1989). 